members in East London have used a Compassionate Community-style approach, acknowledging end of life care as a social process, and everyone's business.

Aim To develop a culturally inclusive, BAME-community-led befriending and advocacy service, working with those who are isolated, terminally ill or reaching end-of-life. The intention is to work in partnership, with a focus on cultural, spiritual and social needs, rather than to replace existing provision.

Methods Since its start in 2013, Eden Care has provided an endof-life service which is open to needy people from all communities. There are currently 35 Eden Care volunteers, most of whom are 'experts-by-experience' after experiencing terminal illnesses alongside family members or friends. 15 of the volunteers have undergone a DBS check and vetting process, and received specialist training to provide high-quality, non-discriminatory and non-judgmental services.

Results 15 people/clients have been paired with a befriender providing support with personal, social and spiritual needs. A Rapid Response Team can carry out 24/7 visits when a person is nearing death. The grassroots, responsive approach means that additional work is now also being done with a support service for Muslim burials; and on giving voice to those nearing end-of-life and the wider community, thereby enhancing the local statutory end-oflife strategy.

Conclusions Clients and families who might be considered 'hard to reach' by statutory providers are served in truly responsive and culturally appropriate ways when communities lead the work.

\section{P-100 THE ADVANCE CARE PLANNING SERVICE}

Kevin Chesters, Michelle Baskerville. Douglas Macmillan Hospice, Stoke on Trent, UK

\subsection{6/bmjspcare-2016-001245.123}

Our hospice has developed a model of advance care planning support that helps patients to explore what they want to do and how to achieve it. This has resulted in their wishes and preferences being met including achieving their preferred place of death.

The model takes a partnership approach between the clinicians at the hospice and the patients they support, helping them consider choices and preferences for future care and treatment. Those clinicians can then refer in to the hospice's advance care planning service where a social work assistant supports the person to write an Advance Statement and record their wishes. The patient holds a copy and anyone else as appropriate and consented to by them. The service supports patients who want to write an Advance Decision to Refuse Treatment as well. They talk to a doctor or nurse who knows them well so to be sure that they understand any potential implications of declining treatments.

The documents are reviewed every six months unless the person wants to make changes before or a review is prompted in some other way. Records indicate when reviews are due and documents are updated and disseminated again if required.

This has proved to be a more fluid and efficient system in providing this kind of support as it relieves the clinicians of the administrative elements of writing, storing and disseminating the documents.

If the patient wants to register a Lasting Power Attorney (LPA) the social work team manager can act as certificate provider for their applications. Although anyone can register LPAs via the Office of the Public Guardian, many people are either referred to, or seek the help of solicitors which can be costly. The service provides equitable access for those are unsure how to register or unable to afford.

\section{P-101 EXPERIENCES OF ADVANCE CARE PLANNING WITH PEOPLE LIVING WITH CHRONIC PROGRESSIVE NEUROLOGICAL CONDITIONS}

${ }^{1,2}$ Lorraine Petersen. ${ }^{~}$ Arthur Rank Hospice Charity, Cambridge, UK; ${ }^{2}$ CUHFT

\subsection{6/bmjspcare-2016-001245.124}

The recent 2016 NICE guidance 'Motor Neurone Disease: Assessment and management' describes a model of multi-disciplinary holistic care. This includes providing opportunities to plan for end-of-life care. The Mental Capacity Act 2005 clarified the legal status of advance care planning but patients need to be in receipt of accurate information to be able to make plans, especially if they have not experienced treatments they might wish to refuse. When the hospice started an initiative to increase access to patients living with neurological conditions a number were referred for advance care planning. At the time (2011) the 'Preferred Priorities for Care' document was in use but staff had little experience in discussing or documenting patients' decisions regarding future refusal of treatments. This took place when patients visited the local Motor Neurone Disease (MND) Care Centre but for patients living with other progressive neurological conditions, it was unclear if they had any opportunities at all. A lead consultant was referred all the patients who might want to write an advance care plan. In order to ensure the resulting document would be 'valid' the consultant created a decision-aid that contained instructions on what details to include. To ensure these decisions were 'applicable' examples of scenarios involving potentially life-saving treatments were laid out for the patient to consider. The decision-aid underwent a number of revisions as exemplars were added to illustrate how the patient might document their decisions.

To date, 18 patients have completed combined Advance Decisions to Refuse Treatment/advance statements. One patient completed but never signed, one documented his decisions informally. The decision-aid, an analysis of the decisions the plans include, how these vary across the different diseases, who supported the patient in writing it, whether they have been revised and reflection on our experiences will be displayed in this poster presentation.

\section{P-102 "4 EASE" - A DEDICATED SELF-MANAGEMENT EDUCATION PROGRAMME}

Jane Finnerty, Kelly De Souza, Carolyn Fillingham. Willowbrook Hospice, Prescot, UK

\subsection{6/bmjspcare-2016-001245.125}

Background The aim of Wellbeing Services is to optimise patients' function, wellbeing and enable them to live independently with the best quality of life. To help achieve this we follow a rehabilitative model to empower patients to live life to their full potential for as long as possible. Education sessions have been designed with self-management as the focus.

Method A 12-week programme is delivered on a rolling basis within Day Hospice which provides a 'tool box' of self-help techniques to help maximise symptom control/quality of life. Sessions 


\begin{tabular}{ll}
\hline Breathlessness management & Physiotherapist \\
\hline Anxiety management part $1 \& 2$ & Complementary Therapist \\
Chair-based exercises & Physiotherapist/Occupational therapist \\
Energy conservation & Occupational Therapist \\
Tai Chi & Holistic Therapist \\
Pain management & Staff Nurse \\
Body image/self-esteem & Complementary Therapist \\
Nutrition & Staff Nurse \\
Creative therapy for well-being & Holistic Therapist \\
Reminiscence through music & Staff Nurse \\
\hline
\end{tabular}

provide opportunity for patients to have input with all members of the multi-disciplinary team. This helps patients to better understand their symptoms, empowering their use of resources and information to their advantage. Visual prompts include the use of an A1 flip chart and handouts to increase inclusion and underpin learning. The sessions provide peer support through informal discussions and can reassure patients that they are not alone; that they can talk openly, relate to each other and share experiences in a safe and reassuring environment.

Education Programme Evaluation The sessions support patients to reinforce existing coping strategies, develop and learn new ones. They aim to empower patients to alleviate and increase well-being. Importantly, the sessions prompt effective self-management of common symptoms experienced by palliative patients.

Conclusions The programme has helped to raise awareness of symptom control and has provided a holistic 'tool box' of self-help techniques to support patients in a personal sense of wellbeing and fulfilment; thus maximising enjoyment and quality of life.

\section{P-103 SELF-MANAGEMENT DVD: SUPPORT FOR PATIENTS IN THE HOME SETTING}

Jane Finnerty, Kelly De Souza, Tony Foster. Willowbrook Hospice, Prescot, UK

\subsection{6/bmjspcare-2016-001245.126}

Background Patients are living longer with a range of life limiting illnesses, the Wellbeing Service have adapted the Day Hospice education programme, and created with the assistance of a volunteer photographer a series of DVDs to help maximise patient's quality of life. Patients can access these free on discharge to enable on-going support at home.

Method The DVDs provide a visual and audio holistic 'tool box' of self-help techniques to reinforce self-management of common symptoms experienced by palliative patients. An audit from the education programme identified the following as key areas for support; breathlessness management, anxiety management, fatigue and mobility.

Topics on the DVDs include:

\begin{tabular}{ll}
\hline Self- massage/relaxed breathing techniques and guided & Complementary \\
visualisation & Therapist \\
Breathing techniques/relaxed positional techniques & Physiotherapist \\
Gentle chair based exercises & Occupational Therapist \\
Chair-based Tai Chi & Holistic Therapist \\
\hline
\end{tabular}

Results The DVDs may help patients to review and recap skills and coping strategies learnt within wellbeing services. They reinforce self-management of symptoms to support and empower patients with their own well-being thus maximising quality of life.

Benefits The DVDs:

- continue to provide reassurance and support at home following discharge

- may increase time between referrals back to service

- continue to empower and guide patients with their self-care

- may help carers to provide additional support to their loved ones' care, by increasing their knowledge and raising their awareness of strategies that patients can use to self-manage their symptoms

- provide a very good visual aid - especially to those that are hard of hearing. It may also be especially helpfully for patients that have short-term memory or impaired cognition.

Conclusions The DVDs provide on-going support post discharge for both the patient and their carers through the application of the techniques learnt at day hospice whilst in the comfort of their own home.

\section{P-104 SITTING DOWN BUT MOVING FORWARD!}

Jenny Sherburn. ellenor, Gravesend, UK

10.1136/bmjspcare-2016-001245.127

Background The opportunity to access exercise classes through the hospice is a more recent phenomenon, challenging the attitudes of staff and patients about the role of rehabilitation and exercise within palliative care. The physical and emotional benefits of exercise are well documented, so in response to this, and patient demand, 'drop in' seated exercise classes were created.

Aims

1. To provide the opportunity to exercise in a safe environment

2. To improve the fitness of the palliative patient group

3. To promote independence and wellbeing within the palliative patient group

4. To raise the profile of physiotherapy.

Approach A one-hour seated exercise session is delivered by a physiotherapist twice a week. Every attendee completes a Timed Up and Go test (T.U.G) prior to starting their first class, this test is repeated every fourth attendance.

Outcomes Classes have run for eight months, in that time 43 people attended. Out of these:

10 were both ambulatory and attended more than four sessions.

The T.U.G results are significant, demonstrating that $100 \%$ of participants achieved a quicker walk time on subsequent T.U.G testing.

Only one subject recorded a slower time on their fourth test. Conclusion These results suggest that participants have achieved an improvement in their fitness, and using the T.U.G as a guideline, $66 \%$ of attendees have reduced their walk time to $<14$ seconds and therefore, could have reduced their risk of falls.

The provision of a weekly exercise group has put physiotherapy 'on the map'. Verbal feedback from patients attending the group has been overwhelmingly positive: "It's made me feel more confident" and as a result the group is becoming more popular. 